ARTICLE

https://doi.org/10.1038/s41467-020-14709-y

\title{
Hierarchically-structured metalloprotein composite coatings biofabricated from co-existing condensed liquid phases
}

\author{
Franziska Jehle (10 ${ }^{1}$, Elena Macías-Sánchez ${ }^{1}$, Sanja Sviben ${ }^{1}$, Peter Fratzl ${ }^{1}$, Luca Bertinetti (iD ${ }^{1 凶} \&$ \\ Matthew J. Harrington (1) 1,2四
}

Complex hierarchical structure governs emergent properties in biopolymeric materials; yet, the material processing involved remains poorly understood. Here, we investigated the multiscale structure and composition of the mussel byssus cuticle before, during and after formation to gain insight into the processing of this hard, yet extensible metal cross-linked protein composite. Our findings reveal that the granular substructure crucial to the cuticle's function as a wear-resistant coating of an extensible polymer fiber is pre-organized in condensed liquid phase secretory vesicles. These are phase-separated into DOPA-rich protogranules enveloped in a sulfur-rich proto-matrix which fuses during secretion, forming the sub-structure of the cuticle. Metal ions are added subsequently in a site-specific way, with iron contained in the sulfur-rich matrix and vanadium coordinated by DOPA-catechol in the granule. We posit that this hierarchical structure self-organizes via phase separation of specific amphiphilic proteins within secretory vesicles, resulting in a meso-scale structuring that governs cuticle function.

\footnotetext{
${ }^{1}$ Department of Biomaterials, Max Planck Institute of Colloids and Interfaces, 14424 Potsdam, Germany. ${ }^{2}$ Department of Chemistry, McGill University, 801 Sherbrooke Street West, Montréal, QC H3A OB8, Canada. ${ }^{\bowtie}$ email: luca.bertinetti@mpikg.mpg.de; matt.harrington@mcgill.ca
} 
T he ability to endow polymeric materials with nano-scale and meso-scale structural hierarchy via self-assembly is an important materials design challenge with implications for tissue engineering, drug delivery and smart polymer engineering $^{1-4}$. Polymer scientists aim to achieve control of multiscale organization through precisely defined chemical structure and engineered supramolecular interactions ${ }^{2,5,6}$, which can be employed for example, as scaffolds for guiding polymerization of materials with defined hierarchy (e.g., mesoporous silica $)^{7-9}$. Similarly, proteins also possess an inherent capacity for supramolecular self-organization-determined by amino acid sequence - that has been harnessed through evolution for fabricating bulk materials/tissues with enhanced function, controlled through multiscale hierarchical structure ${ }^{10-15}$. Therefore, elucidating the physical and chemical underpinnings of biological material assembly provides a rich source of inspiration for designing bottom-up processes to fabricate hierarchically structured, functional polymeric materials.

The mussel byssus is a hierarchically structured protein-based material that has emerged as an exciting model system for understanding supramolecular assembly of complex material structures under sustainable processing conditions (Fig. 1) ${ }^{11,13}$. Mussels fabricate a byssus as an attachment holdfast for anchoring on rocky surfaces in seashore habitats (Fig. 1a), where they face forces from crashing waves ${ }^{16}$. Each byssal thread is covered by a thin protective cuticle at its distal end (i.e., away from the mussel) (Fig. 1), which is highly extensible, yet stiff and hard ${ }^{17-21}$. These typically opposing properties likely function to protect the stretchy fibrous core within and originate from the composite-like structure of micron-sized inclusions known as granules embedded in a continuous matrix ${ }^{18}$ (Fig. If-h). While it was originally proposed that the granules are hard inclusions in a softer matrix ${ }^{17,18}$, more recent results suggest that granules function to retain water at low hydration, behaving softer than the matrix under dry condition ${ }^{21}$. Regardless, nanoindentation experiments indicate that the cuticle acquires nearly $85 \%$ of its stiffness/hardness from metal coordination cross-links between metal ions (such as $\mathrm{Fe}$ and $\mathrm{V}$ ) and 3,4-dihydroxyphenylalanine (DOPA), a post-translational modification of Tyrosine elevated in the cuticle protein, mussel foot protein 1 (mfp-1) (Fig. 1$)^{20}$. Notably, the DOPA-metal interactions are more concentrated in the granules than the matrix ${ }^{17}$. (n.b. species specific forms of mfp-1 are named according to the species; e.g., mefp- 1 for Mytilus edulis)

Numerous reviews highlight recent efforts to mimic the DOPAmetal cross-link strategy with mussel-inspired catechol-functionalized polymers ${ }^{22-25}$; yet, none of these materials reproduces the complex hierarchical structure of the cuticle or its properties. Indeed, little is understood about how the nanostructure of the cuticle and granules is achieved. It is known that the proteins that comprise the cuticle are stored within secretory vesicles in the mussel foot-the organ responsible for synthesizing and stockpiling the proteins that make the byssus (Fig. $1 \mathrm{~b}, \mathrm{c})^{13,26,27}$. Selfassembly of the cuticle occurs within minutes via release of vesicle contents into a groove running along the foot, during which they coalesce and spread over the already formed collagenous core of the byssus fiber (Fig. 1b-e) ${ }^{13}$. However, many questions remain concerning how the intricate cuticle substructure emerges via selfassembly and how metal ions are infiltrated to cure the formed cuticle.

Here, we report an in-depth compositional and ultrastructural investigation of the structure and self-assembly of the mussel byssus cuticle. Specifically, we investigate the 3D nanostructure and elemental composition of the secretory vesicles and the cuticle itself, utilizing a combination of focused ion beam scanning electron microscopy (FIB-SEM), transmission electron microscopy (TEM) and scanning transmission electron microscopy with energy dispersive X-ray spectroscopy (STEM-EDS). By examining the different stages of assembly, we gain important new insights into the formation process and function of this complex biological material, with bearing on the design of technically and biomedically relevant composite materials.

\section{Results}

Nanostructural investigation of cuticle secretory vesicles. Previous investigations have identified that the precursor proteins forming the cuticle are stored in secretory vesicles in the mussel foot within a region known as the enzyme or cuticle gland (Fig. 1d) ${ }^{13,26}$. Transmission electron microscopy (TEM) imaging of post-stained thin sections of the cuticle gland of Mytilus edulis revealed that each micron-sized secretory vesicle possesses at least two distinctive regions-a darker outer phase (more heavily stained with Os) and a lighter, less-stained inner phase (Fig. 2a). The distinctive biphasic brain-like texture of the inner phase consisting of lighter stained connected layers was previously observed in cuticle secretory vesicles of another species, Mytilus galloprovincialis ${ }^{26}$, and is highly reminiscent of native thread cuticle granule structure (Fig. 1h)-leading us to name this the proto-granule and the outer phase, the proto-matrix. A third, very lightly stained crescent-shaped phase is also observed at the outer periphery of most cuticle secretory vesicles. FIB-SEM of chemically fixed foot tissue samples enabled 3D rendering of secretory vesicles in a small region of the cuticle gland close to the secretion point, revealing a tightly packed arrangement of nearly spherical vesicles (Fig. 2b, c). Consistent with TEM, there are at least three discernible phases in these vesicles that stain differently, corresponding to the proto-granule, proto-matrix and crescent phase (Fig. 2b-d). Volumetric analysis of the 3D data of 28 individual cuticle secretory vesicles indicates similar distribution of volume fractions of the three phases in all vesicles suggesting a highly regulated formation process (Supplementary Fig. 1).

Compositional investigation of cuticle secretory vesicles. Compositional analysis of cuticle secretory vesicles was performed with STEM-EDS, revealing an elevated and uniform nitrogen content in cuticle secretory vesicles compared with the surrounding intracellular material (Fig. 2e). In contrast, there is an approximately two to three-fold higher sulfur content in the proto-matrix relative to the proto-granules (Fig. 2e, f). Notably, recent transcriptomic studies have identified a family of putative cysteine-rich proteins (mfp-16-mfp-19) within the cuticle gland of a related species Mytilus californianus ${ }^{28}$. This is consistent with previous cytochemical studies predicting the presence of cysteinerich proteins within the cuticle secretory vesicles of Mytilus galloprovinicialis ${ }^{26}$. The fact that the proto-granule contains a lower sulfur signal substantiates the previous supposition that the granules mainly contain mefp-1, which completely lacks cysteine ${ }^{17}$. Furthermore, cysteine is a strong target of $\mathrm{OsO}_{4}$ staining 29,30 , which helps explain the contrast observed in TEM and FIB-SEM. Notably, within the sensitivity limit, we did not detect peaks indicative of transition metal ions (e.g., Fe and V) in the EDS spectra from unstained cuticle secretory vesicles (Supplementary Fig. 2), even though these metals were previously detected in the mature cuticle ${ }^{17,19,20}$. This result is consistent with recent findings suggesting that metal ions are added into the cuticle in a post-secretion curing step ${ }^{13}$.

Structural investigation of cuticle formation. To investigate the dynamic transition from the storage phase of cuticle secretory vesicles into a hard and flexible mesostructured coating, TEM was performed on vesicles secreted into the foot groove. Vesicle 

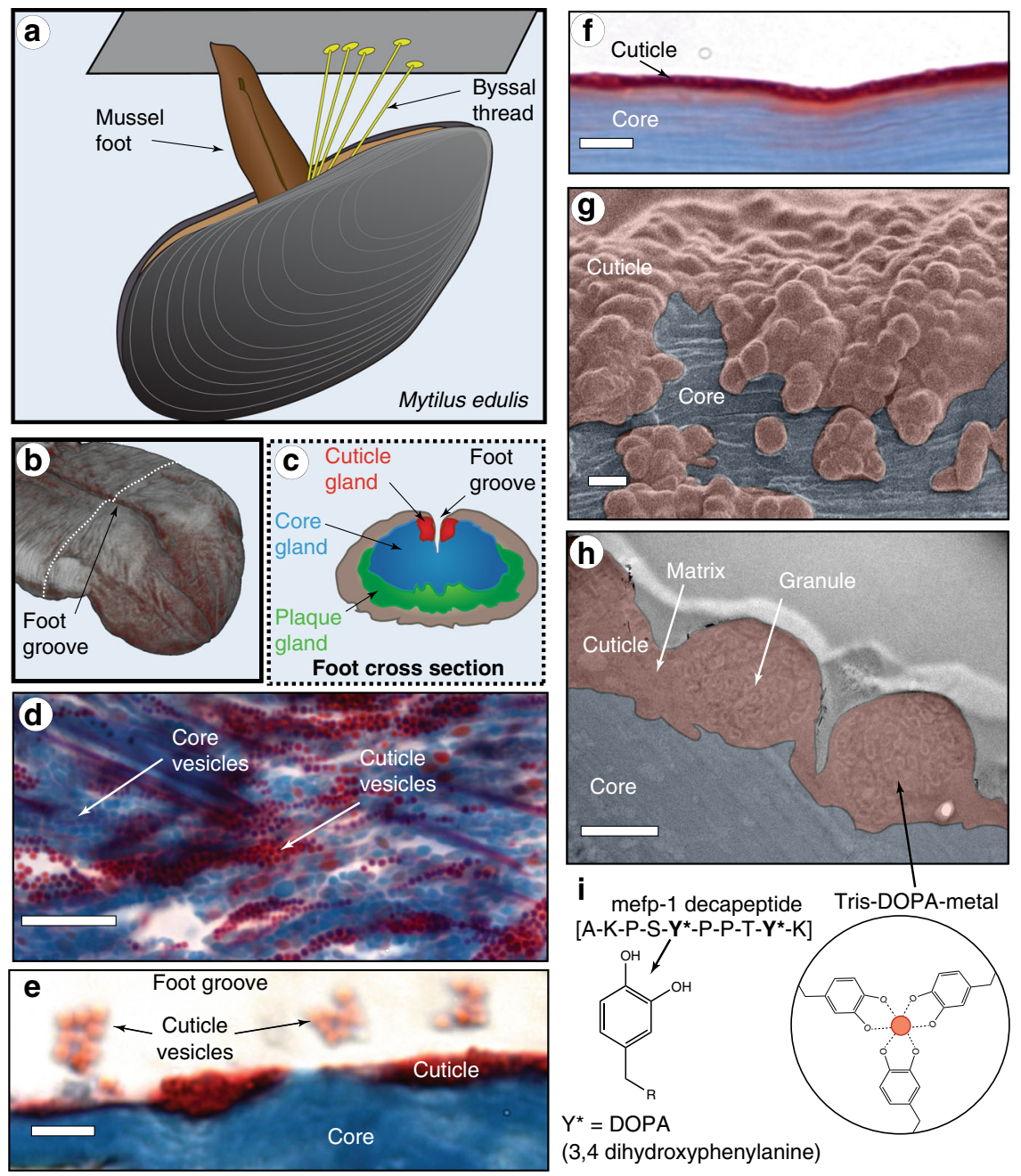

Fig. 1 Overview of byssus cuticle formation and structure. a Marine mussels (Mytilus edulis) synthesize byssal threads using an organ known as the foot. b CT image of the distal region of a mussel foot highlighting the foot groove, in which the thread forms. c Schematic of a foot transverse cross-section from a region of the foot indicated by white dashed line in $\mathbf{c}$ showing location of specific glands in which thread-forming proteins are stockpiled. $\mathbf{d}$ Trichrome stained transverse section of foot gland tissue showing the core (blue) and cuticle (red) secretory vesicles. Scale bar $=10 \mu \mathrm{m}$. e Trichrome stained longitudinal thread section captured during induced formation showing the core and formation of the cuticle. Clusters of cuticle secretory vesicles coalesce and are partially spread over the core surface creating the cuticle. Scale bar $=4 \mu \mathrm{m}$. $\mathbf{f}$ Trichrome stained longitudinal section of a native distal byssal thread fixed on a glass slide. Scale bar $=4 \mu \mathrm{m}$. $\mathbf{g}$ SEM image of a native distal thread surface with false coloring to differentiate the cuticle (red) and exposed core (blue). Scale bar $=1 \mu \mathrm{m}$. $\mathbf{h}$ TEM image of a thin osmium stained transverse cross-section of a native distal byssal thread with false coloring to indicate the cuticle and fibrous core. Scale bar $=500 \mathrm{~nm}$. $\mathbf{i}$ The cuticle is known to be partially comprised of a protein called mefp-1, with an extended domain made of decapeptide repeats containing 3,4-dihydroxyphenylalanine (DOPA), which is believed to be coordinated to metal ions including vanadium and iron. Panels b, $\mathbf{d}$-f are adapted from ref. ${ }^{13}$ under the Creative Commons License.

secretion was induced by injection of $0.56 \mathrm{M} \mathrm{KCl}$ into the pedal nerve of the foot, as previously used to study thread formation ${ }^{13,31}$. TEM images of the distal region of induced threads chemically fixed several minutes into the formation process reveal that cuticle secretory vesicle contents fuse and coalesce already within the secretory ducts leading from the glands to the foot groove (Fig. 3a). This reveals that the protomatrix retains a liquid-like behavior as it coalesces with the contents of other vesicles, showing no apparent boundaries. However, the proto-granules remain as separate entities with the biphasic brainy structure intact. Within the groove, the coalesced secretory vesicles spread over the collagenous core forming a thin layer, as previously shown with histology and confocal Raman spectroscopy (Figs. 1e, $3 \mathrm{~b}, \mathrm{c})^{13}$. This granular morphology is very similar to the native cuticle structure (Fig. $1 \mathrm{~h}$ ). Taken together with the TEM-EDS measurements (Fig. 2e, f), these observations suggest that the cuticle secretory vesicles contain two immiscible protein phases represented by the proto-matrix and protogranule that are pre-organized within the vesicles to facilitate rapid assembly of a hierarchically structured coating.

Nanostructural investigation of native cuticle. The nanoscale structural and compositional details of the native cuticle were next investigated with FIB-SEM and STEM-EDS on chemicallyfixed distal thread samples, both of which revealed that M. edulis thread cuticles consist of a single layer of granules, rather than the several layers observed in other Mytilus species ${ }^{18,21}$. FIB-SEM analysis enabled the visualization of the matrix and granules, as well as the convoluted brain-like internal substructure of the granules (Fig. 4a, b). 3D reconstruction of the image stacks revealed the connectivity of the matrix phase and heavily stained part of the granule in three dimensions and further indicates that the lightly stained (darker) region within the granules is 

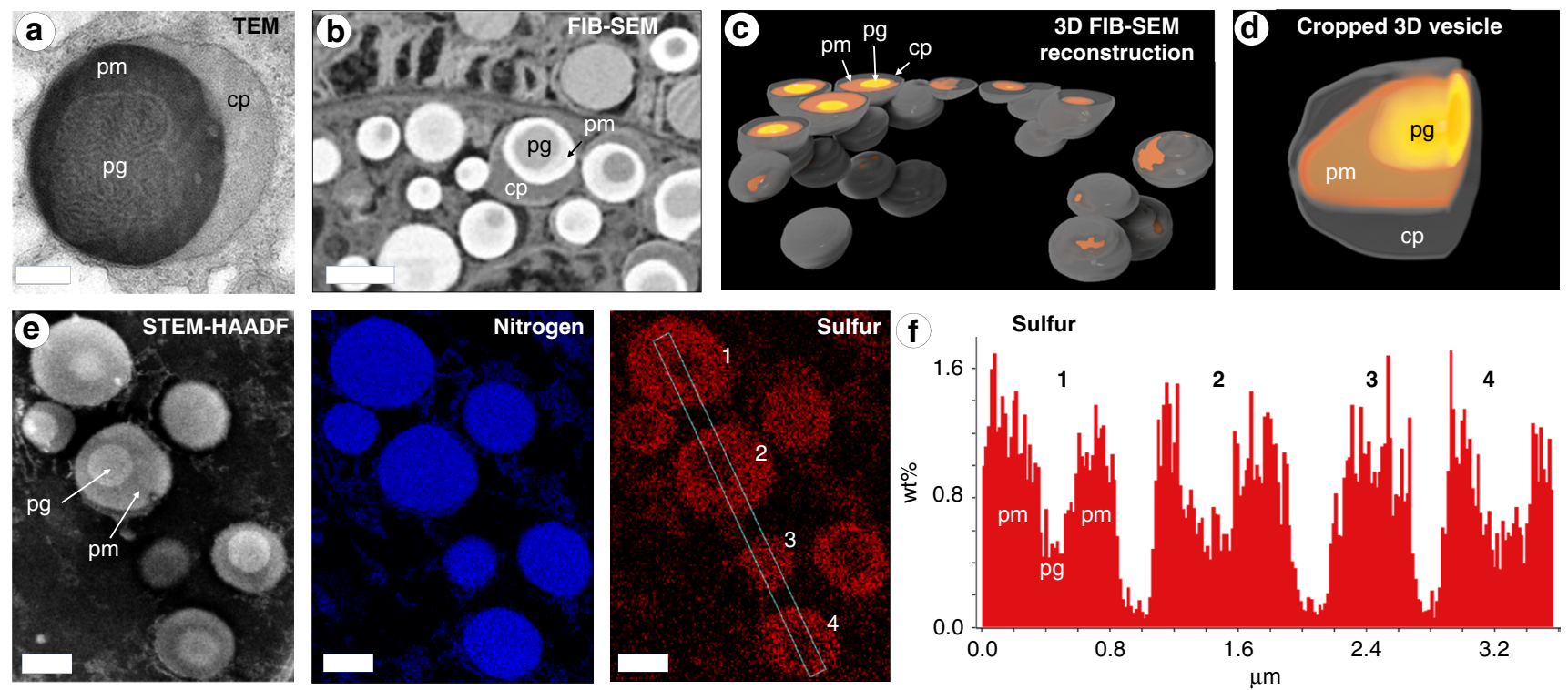

Sulfur

Fig. 2 TEM and FIB-SEM imaging of cuticle secretory vesicles in mussel foot tissue. a TEM image of osmium stained cuticle secretory vesicle from region similar to Fig. 1d. Contrast is achieved by different osmium staining with the proto-matrix (pm) staining the heaviest, the crescent phase (cp) staining the lightest and the proto-granules (pg) staining in between. Scale bar $=200 \mathrm{~nm}$. b FIB-SEM image of osmium stained region of cuticle gland similar to Fig. 1d. Contrast is inverted compared to TEM, but pg, pm and cp can clearly be differentiated. Scale bar $=1000 \mathrm{~nm}$. c Using electron density contrast, secretory vesicles and their inner structure were reconstructed in 3D from an image stack consisting of 392 images. d Magnified and cropped 3D image of a cuticle secretory vesicle showing all three phases. e STEM-EDS compositional analysis of native cuticle granule and matrix regions, showing distribution of nitrogen and sulfur in the region in the STEM-HAADF image in the left panel. Scale bar $=500 \mathrm{~nm}$. $\mathbf{f}$ Relative sulfur wt\% (not calibrated) collected from a transect across four secretory vesicles in dotted box in e reveals that pg has approximately one half the amount of sulfur as pm.

comprised of a connected network of flattened layers resembling a bicontinuous phase of a micro phase-separated block copolymer (Fig. $4 \mathrm{c}, \mathrm{d})^{32}$. Image analysis reveals the flattened layers of multiple granules within a given thread possess a highlydefined thickness of $\sim 20 \mathrm{~nm}$, although this varied between different threads (Fig. 4d and Supplementary Fig. 3; Supplementary Table S1). Additionally, the flattened layers within the granules are oriented along a common direction approximately $45^{\circ}$ to the fiber axis (Fig. 4c) - the orientation of which is consistent between different granules within the same thread. The 3-dimensional (spherically averaged) autocorrelation functions of the different measured granules are similar to each other within a given thread, indicating a high homogeneity in their structural features (Supplementary Fig. 3).

Compositional investigation of native cuticle. At first glance, STEM-EDS compositional studies of the mature cuticle are largely consistent with those measured on the cuticle secretory vesicles. Similar to the proto-granules and proto-matrix in the vesicles, the granules and matrix have uniform nitrogen levels, while the matrix phase has a significantly higher sulfur signal than the granules (Fig. 4e, S4). However, while transition metal ions were not detected in the cuticle secretory vesicles (Supplementary Fig. 2), both Fe and V were detected by EDS in the native cuticle (Fig. 4e). Unexpectedly, however, metal distribution was micro-partitioned within the cuticle, with $\mathrm{V}$ explicitly associated with the granules and Fe associated only with the matrix with a sharp interface at the boundary. This is consistent with previous Raman spectroscopic measurements across different Mytilid species showing that DOPA-V coordination is predominantly detected in native cuticles, rather than DOPA-Fe coordinationalthough it should be noted that Fe can be artificially introduced and coordinated following removal of $\mathrm{V}$ with EDTA ${ }^{17,20}$. Because the metals are not present in the secretory vesicles, this finding also implies that the metal ions spontaneously segregate between the matrix and granules when introduced during distal thread formation.

\section{Discussion}

The findings of this study suggest that the granular mesostructure of the cuticle is achieved through a membrane-bound liquid-liquid phase separation (LLPS) consisting of immiscible fluid protein phases. Co-existing condensed liquid protein phases are observed in nucleolar subcompartments and believed to be important for tuning the vectorial transport and processing of rRNA $^{33}$. In contrast, within the cuticle secretory vesicles, the coexisting LLPS leads to a micro-scale to nano-scale distribution of proteins with different functional groups (i.e., DOPA and Cysteine) that contribute to cross-linking the liquid phase to solid when triggered during assembly. This effectively tunes the viscoelastic properties of the final material at the mesoscale. Based on our current understanding of the role of metal coordination complexes in the byssus cuticle, we posit that microscale partitioning of specific metal coordination cross-links between the granules and matrix has important implications for the dynamic properties of this material, and it is important to understand why and how this occurs. Current models postulate different mechanical properties in the matrix and granules-either hard granular inclusions in a soft matrix ${ }^{17,18}$ or soft, water-absorbent granules in a stiffer matrix (at least under low hydration conditions) ${ }^{21}$. However, both models rest on results from quasistatic mechanical indentation performed at a single loading rate, which do not access the dynamic nature of the metal coordination bonds. On the other hand, rheological investigations of mussel-inspired catechol-enriched metallopolymers have demonstrated clearly that DOPA-V coordination bonds possess a more than 10-fold longer bond lifetime (and thus, slower relaxation time) than DOPA-Fe bonds $s^{34}$. This is functionally relevant considering that mussels face an enormous range of mechanical loading rates from rapid crashing waves with velocities up to $30 \mathrm{~m} / \mathrm{s}$ to the extremely slow 
Thread formation
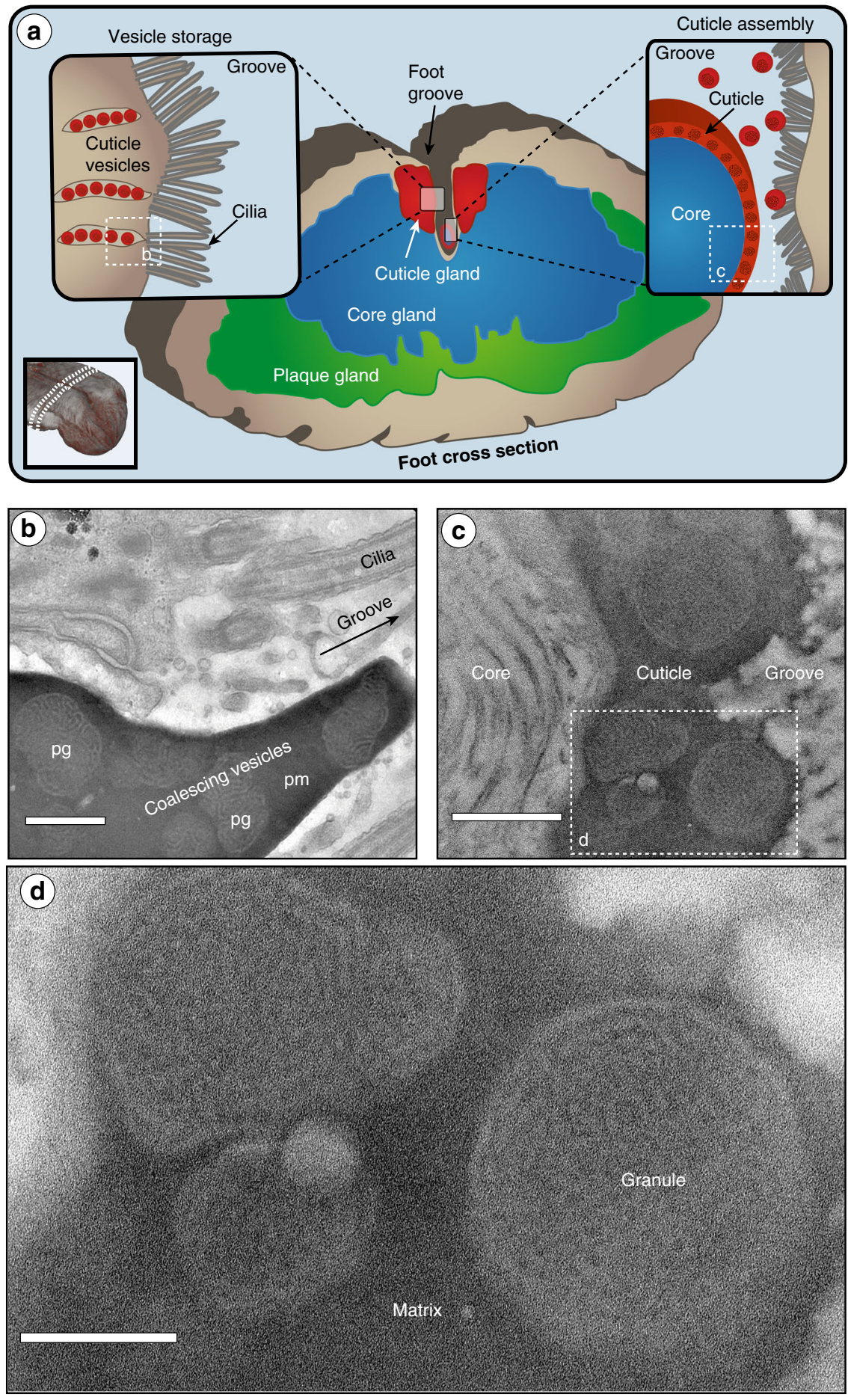

Fig. 3 Cuticle formation in the mussel foot groove via coalescence of cuticle secretory vesicle contents. a Schematic overview of transverse section of mussel foot tissue showing anatomy of byssal glands. Cuticle secretory vesicles (in red) are lined up at the edge of the cuticle gland, ready to be secreted into the groove, aided by cilia. During cuticle assembly, the secretory vesicle contents cluster and coalesce into the groove spreading over the formed core surface. $\mathbf{b}$ TEM image from a stained section of an induced foot in the region indicated in $\mathbf{a}$, showing coalesced cuticle secretory vesicles about to be released into the groove. Notably, the proto-matrix (pm) of several vesicles merges to form a continuous matrix, while the proto-granules (pg) retain their brain-like structure. Scale bar $=500 \mathrm{~nm}$. c TEM image from stained section of an induced foot in the region indicated in a, showing a formed induced thread with intact cuticle. Scale bar $=500 \mathrm{~nm}$. d Magnified region from c showing details of the induced cuticle including granule and matrix. Scale bar $=200 \mathrm{~nm}$.

loading from predators such as sea stars ${ }^{16}$. Thus, it seems plausible that the physical demands of life in the intertidal have led to evolution of an adaptive coating that responds to a wide range of loading conditions (e.g., strain rates, extensions, and forces), achieved through hierarchical organization of dynamic bonds.
Fabricating this functional composite structure reproducibly requires a remarkable degree of process control during assembly, which is apparently achieved by pre-organizing the various cuticle components into co-existing phase-separated liquid phases within the secretory vesicles prior to assembly (Fig. 2). Until recently, 

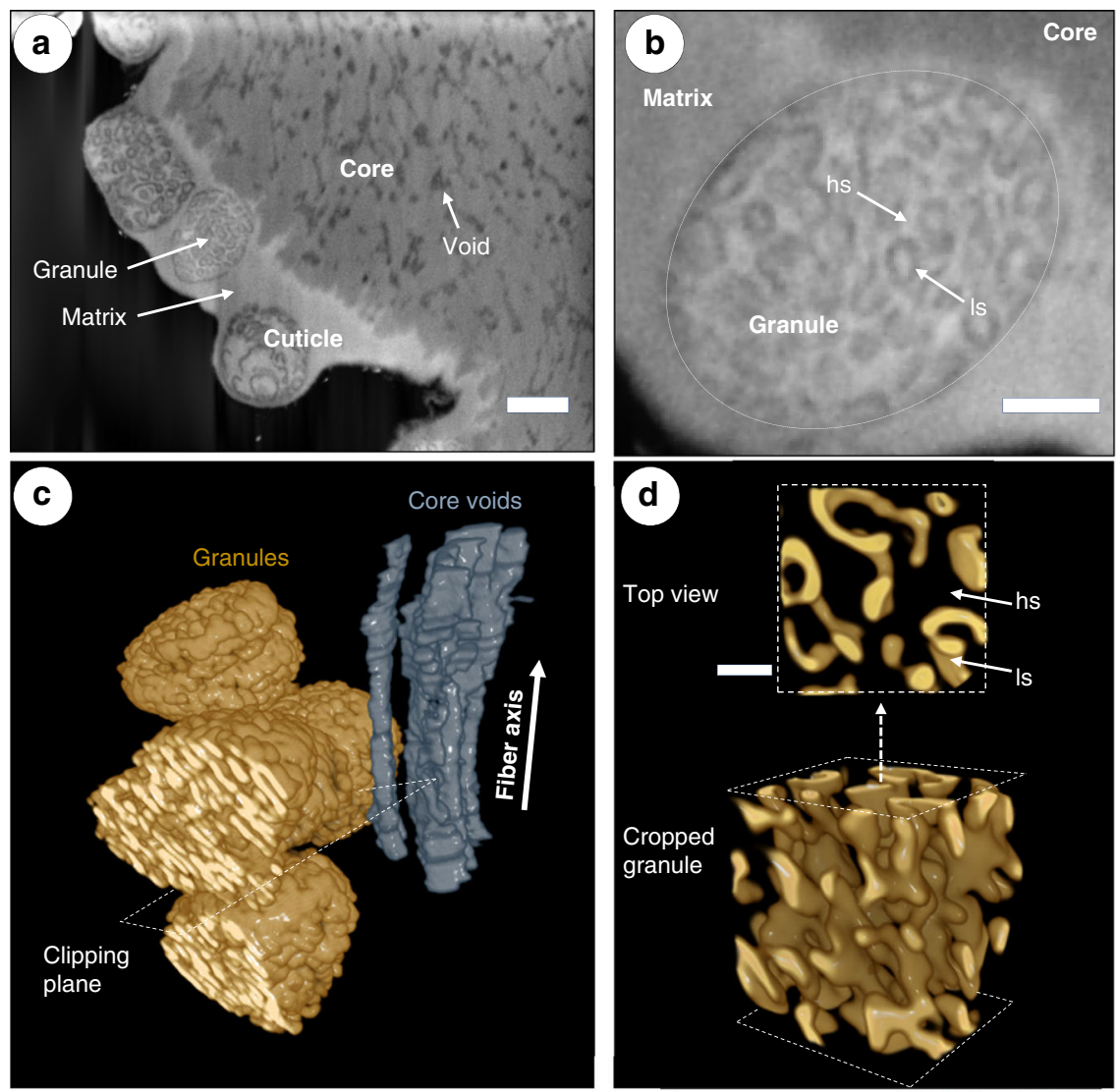

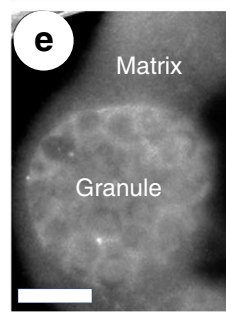

STEM HAADF

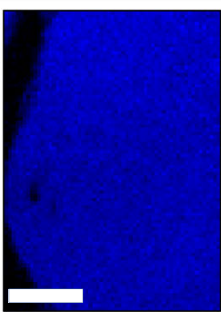

Nitrogen

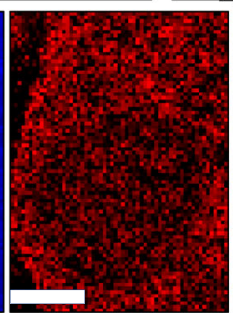

Sulfur

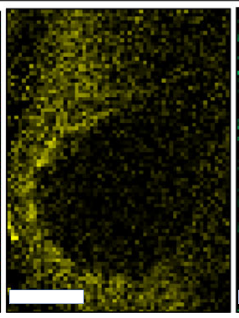

Iron

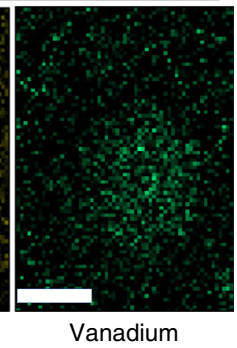

Fig. 4 FIB-SEM 3D-reconstruction of intragranular nanostructure in native thread cuticle. a FIB-SEM image of osmium stained region of native distal thread showing substructure of the core and cuticle, including granule and matrix in the cuticle and previously described voids in the core. Scale bar $=500 \mathrm{~nm}$. b Higher magnification FIB-SEM image of a single granule. Contrast arises from heavily staining (hs) and lightly staining (Is) regions within a single granule. n.b. contrast is inverted compared to TEM, with elements of higher atomic mass appearing brighter. Scale bar $=200 \mathrm{~nm}$. c 3D reconstruction of FIB-SEM image stack using the contrast of the lightly staining (Is) phase of the granules and the core voids (which are parallel to the fiber axis). d Granule cropped using clipping planes parallel to the one indicated in c revealing internal structure of the Is phase, consisting of bicontinuous flattened layers with a thickness of $\sim 20 \mathrm{~nm}$. The transparent region between layers constitutes the heavily staining (hs) phase from $\mathbf{b}$. Scale bar $=80 \mathrm{~nm}$. e STEM-EDS compositional analysis of native cuticle granule and matrix regions, showing distribution of nitrogen, sulfur, iron, and vanadium in the region in the STEM-HAADF image on the left. Scale bar $=100 \mathrm{~nm}$.

only DOPA-rich mefp-1 was confirmed to be present in the cuticle (Fig. 1i). However, as mentioned, transcriptomics has identified four new putative cuticle proteins (mfp-16-mfp-19) in a closely related species ${ }^{28}$, all of which are enriched in cysteine and likely present primarily in the proto-matrix/matrix, based on our STEM-EDS findings and previous cytochemical evidence ${ }^{26}$. The rapid coalescence of the proto-matrix into a solid material during formation is likely related to the well-known ability of cysteine to participate in various covalent and non-covalent interactions ${ }^{35}$. However, as previously proposed, cysteines in byssus proteins likely also perform a crucial role in redox cycling of the DOPA catechol moiety ${ }^{36}$. Both putative roles will be discussed later in more detail. Evidence that mefp-1 is localized in the granule comes from the previous Raman-based localization of DOPA- $V$ interactions in the granules ${ }^{17,20}$, the different susceptibilities of the granule to chymotrypsin vs. pepsin digestion in cytochemical studies ${ }^{26}$ (Supplementary Fig. 5) and the lower sulfur content in the granules observed here with STEMEDS (Figs. 2e, f, 4e).

Given these compositional findings, what is then the source of complex nano-structure observed in the secretory vesicles and thus, the final cuticle structure? We propose that the bicontinuous structure and lower sulfur signal of granules arises from the immiscibility of mefp-1 and the Cys-rich protein, which is driven by the amphiphilic block co-polymer-like structure of mefp-1 (Fig. S6). Mefp-1 consists of numerous repeats of the decapeptide consensus motif [AKPSYPPTYK] $]_{n}$ in which the tyrosine $(\mathrm{Y})$ residues are largely converted to DOPA (total content of $10-15$ mol\%) and known to interact with metal ions (Fe, V) (Figs. 1i, 5 and Supplementary Fig. 6) ${ }^{17,20}$. The repetitive region of mefp-1 


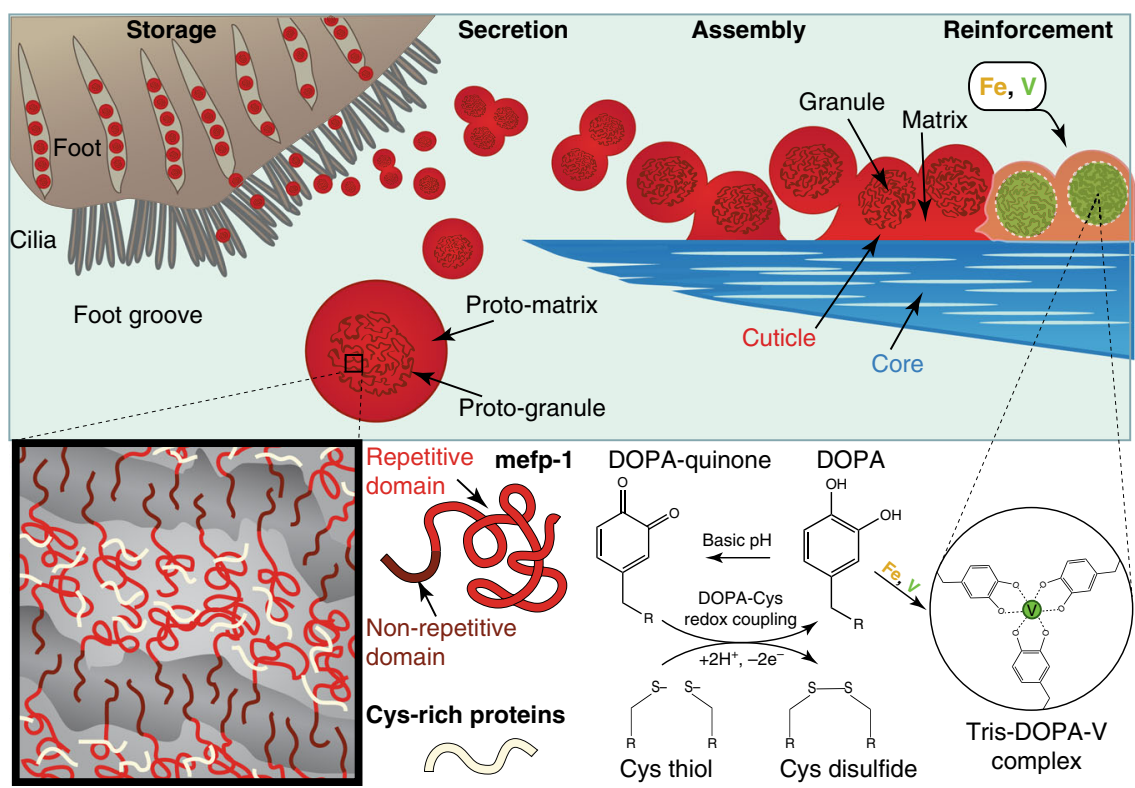

Fig. 5 Schematic model of cuticle assembly via co-existing condensed liquid phase vesicles. To achieve the high degree of compositional, structural and mechanical hierarchy observed in the native byssus cuticle, mussels store the protein precursors as a LLPS of two co-existing phases. The main protein component mfp-1 and the Cys-rich proteins (mfp-16-19) are immiscible due to the amphiphilic nature of mfp-1, leading to phase separation into a bicontinuous structure that characterizes the proto-granule. During secretion and cuticle assembly, the proto-matrix of nearby secretory vesicles fuses forming the continuous matrix of the cuticle, possibly cross-linked via cysteine residues. The intricate nanostructure of the proto-granule is maintained in the newly formed cuticle and contains a much higher local concentration of DOPA than the surrounding matrix. Thus, when metals are added to the thread in a secondary curing, the metal ions that have the highest stability complex with DOPA (i.e., vanadium) diffuse into the granule where they become concentrated relative to iron. This results in different viscoelastic behavior of the granule and matrix, which is likely an adaptive function to life on the highly dynamic rocky seashore. Cysteine may play a secondary, but equally important role as a reducing agent that counteracts the spontaneous oxidation of DOPA to DOPA-quinone under basic conditions, which enables strong metal coordination cross-link formation.

possesses a hydrophilic character due to the high content of conserved Lysine $(\mathrm{K})$ residues. Recombinant expression of truncated mfp-1 consisting of 12 or 22 repeats of the hydrophilic decapeptide have been demonstrated to undergo spontaneous LLPS under high salt conditions mediated via pi-cation interactions ${ }^{37}$, offering support to the coacervate-like nature in the cuticle secretory vesicles. However, at the $\mathrm{N}$-terminus of native mefp-1 and mgfp-1 from M. galloprovincialis is an 60-80 amino acid non-repetitive domain that is markedly less hydrophilic, giving the overall protein an amphiphilic profile (i.e., block copolymer-like) (Fig. 5 and Supplementary Fig. 6). We propose here that the non-repetitive domain provides the impetus for formation of a bicontinuous phase (also observed in M. galloprovincialis), forming the well-defined $\sim 20 \mathrm{~nm}$ thick layers characteristic of the granules, while the positively charged DOPArich phase interacts with the cysteine-rich proteins to produce the heavily stained phase of variable thickness between the flattened layers (Fig. 5). The fact that osmium has a very high affinity to cysteine under the alkaline staining conditions supports this model $^{29,30}$. The matrix on the other hand, seems to consist mostly of the cysteine-rich proteins.

Based on this new model of how the observed meso-structures and nano-structures form in the cuticle secretory vesicles, we consider next how the condensed fluid phase within the vesicles suddenly transitions into a hard, yet stretchy composite material. Our TEM investigation of induced thread formation indicates that the cysteine-rich proto-matrix fuses during assembly, creating the continuous matrix of the cuticle in which the preassembled granules are embedded. As mentioned, cysteine is a highly promiscuous cross-linker with the ability to form covalent bonds via disulfide linkages ${ }^{35}$ or through oxidative cross-linking with DOPA residues ${ }^{36}$. In fact, 5-cysteinyl-DOPA was previously purified from byssus material ${ }^{38}$. However, considering that nearly $85 \%$ of cross-links in the cuticle depend on metal ions ${ }^{20}$, cysteine-based covalent bonds are not the dominant cross-linking mechanism in the matrix. Based on the colocalization of cysteine and $\mathrm{Fe}$ in the matrix, we find the possibility that sulfur-Fe interactions may contribute as loadbearing cross-links highly compelling. While typically found in enzymes involved with redox pathways in cells, recent AFM single molecule force spectroscopic reveal that $\mathrm{Fe}-\mathrm{S}$ clusters can function as reversible sacrificial cross-links in proteins with mechanical breaking force comparable to other metal coordination complexes ${ }^{39}$.

A secondary, but equally important role of the cysteine residues within the granules may be as a reducing agent to stall spontaneous DOPA oxidation in the basic environment of the ocean (Fig. 5). Another cysteine-rich protein called mfp-6 was previously identified in the adhesive secretion of the byssus ${ }^{38}$ and was shown in vitro to prevent DOPA from spontaneously oxidizing to DOPA-quinone (a less effective adherent) through redox cycling ${ }^{36}$. Similarly, DOPA-quinone is inefficient at forming strong metal coordination complexes ${ }^{40}$. Considering that at least $85 \%$ of cross-linking in the cuticle is based on metal coordination bonds ${ }^{20}$, the proposed reducing role of cysteine may be crucial to achieving the dynamic properties, and is likely enhanced by the proposed co-localization with the DOPA-rich repetitive domain of mefp-1 in the granules (Fig. 5).

Shortly after the cuticle forms during assembly via fusion of the proto-matrix, transition metal ions ( $\mathrm{Fe}$ and $\mathrm{V}$ ) are apparently added, providing secondary mechanical reinforcement via metal coordination bonding ${ }^{13,17,20}$. Currently, it is unclear if metal addition is an active or passive process. Cuticles stripped of metal with the metal chelator EDTA are able to uptake metal passively 
from millimolar metal chloride solutions ${ }^{17,20}$. However, pulsechase studies have clearly shown that radiolabeled Fe is actively taken up by mussels and is stored temporarily in the soft tissue from where it is transferred into the byssus fibers over a period of several days ${ }^{41}$. Regardless of the source of the metal ions, Raman spectroscopic investigation of the cuticle revealed that DOPA is able to bind both $\mathrm{V}$ and $\mathrm{Fe}$ within the cuticle when exposed to only the individual ions during in vitro experiments ${ }^{17,20}$. However, all Raman spectra reported for native byssal threads across several species are dominated by the DOPA-V signal, rather than DOPA-Fe ${ }^{17,20}$. It is tempting to propose that this partitioning may reflect the inherent differences in dissociation rates of the DOPA-Fe and DOPA-V complexes previously revealed by rheological studies of mussel-inspired catechol-functionalized hydrogels, showing that DOPA-V has more than an order of magnitude longer bond lifetime than DOPA-Fe complexes ${ }^{34}$. The slower bond dissociation of DOPA-V implies that it will be less likely to exchange with Fe once coordinated, leading to its concentration in the mfp-1-enriched granules over time through dissociation and diffusion. However, the longer bond lifetime, not only affects the distribution of metals, but as already pointed out, will also determine local viscoelastic relaxation behavior defined by the compartmentalization of $\mathrm{mfp}-1$ within the granules. Taking for granted that varying the viscoelastic response on the mesoscale is important to the function of the cuticle (e.g., being hard and extensible, retaining water in dry conditions and/or responding to a range of loading rates ${ }^{17,18,20,21}$ ), this assembly mechanism provides a degree of control over dynamic mechanical response that is not possible in current engineered polymers. Indeed, mimicking this fabrication process and thus, this remarkable degree of control over soft matter response could inspire the design of new responsive smart polymers for a range of applications from flexible electronics and actuated structures to drug delivery and dynamic tissue scaffolds.

\footnotetext{
Methods

Materials. Blue mussels ( $M$. edulis) purchased from the Alfred-Wegener-Institut were maintained at $\sim 14^{\circ} \mathrm{C}$ in an aquarium with artificial salt water. Native threads were harvested from mussels upon arrival and were stored at $4{ }^{\circ} \mathrm{C}$ in water prior to use. Investigations were performed either on native byssal threads $(5-8 \mathrm{~cm}$ in length) or on the foot organ of adult mussels removed with a scalpel $(n=2)$. To investigate cuticle assembly, protein secretion was induced by injecting a small volume of $0.56 \mathrm{M} \mathrm{KCl}$ solution in the base of the mussel feet ${ }^{13,31}$. Induced mussel feet were dissected after at $\sim 5 \mathrm{~min}$ following $\mathrm{KCl}$ injection to investigate vesicle secretion and after $\sim 20 \mathrm{~min}$ to investigate the induced thread cuticle structure. We have complied with all relevant ethical regulations for testing and research of Mytilus edulis.
}

Chemical fixation and embedding. Dissected feet (induced or not) were carefully rinsed with cold water, blotted with a paper towel to remove mucus and pre-fixed for $30 \mathrm{~min}$ at $4{ }^{\circ} \mathrm{C}$ in $3 \%$ glutaraldehyde, $1.5 \%$ paraformaldehyde, $650 \mathrm{mM}$ sucrose in $0.1 \mathrm{M}$ cacodylate buffer $\mathrm{pH}$ 7.2. The foot tissue was then cut into thin crosssections comprising the groove and part of the gland tissue and then fixed for $2 \mathrm{~h}$ at $4{ }^{\circ} \mathrm{C}$ in the same buffer as above. Fixed samples were rinsed $5 \times$ with $0.1 \mathrm{M}$ cacodylate buffer, $\mathrm{pH} 7.2$ at $4{ }^{\circ} \mathrm{C}$ and post-fixed with $1 \% \mathrm{OsO}_{4}$ for $1 \mathrm{~h}$ at $4{ }^{\circ} \mathrm{C}$. Tissue samples prepared for elemental analysis were not treated with $\mathrm{OsO}_{4}$. Samples were rinsed again in $0.1 \mathrm{M}$ cacodylate buffer $\mathrm{pH} 7.2\left(3 \times 5 \mathrm{~min}\right.$ at $\left.4{ }^{\circ} \mathrm{C}\right)$, followed by series dehydration in ethanol $(50 \%, 70 \%, 90 \%, 3 \times 100 \%)$ for $10 \mathrm{~min}$ each step at RT. Dehydrated samples were embedded either in low viscosity Spurr's resin (Electron Microscopy Sciences, \# 14300) for TEM/STEM-EDS or in Hard Plus resin 812 (Electron Microscopy Sciences, \# 14115) for FIB-SEM and polymerized at $70{ }^{\circ} \mathrm{C}$ for at least $48 \mathrm{~h}$. Ultrathin sections of $100 \mathrm{~nm}$ for TEM investigations were prepared using a PowerTome Model XL ultramicrotome (Boeckeler Instruments, Inc.) and mounted on carbon coated $\mathrm{Cu}$ grids (200 mesh) for imaging and on Lacey carbon coated $\mathrm{Cu}$ grids (200 mesh) for EDS measurements. In order to reveal the internal structure of the protogranule, some grids were post-stained with $2 \%$ uranyl acetate for $10 \mathrm{~min}$.

The distal region of native threads were washed $3 \times 5 \mathrm{~min}$ in cold double distilled $\mathrm{H}_{2} \mathrm{O}$ and cut into small pieces of $\sim 3 \mathrm{~mm}$ in length. Fixation was carried out for $1 \mathrm{~h}$ at $4{ }^{\circ} \mathrm{C}$ in $2.5 \%$ glutaraldehyde and $1.5 \%$ paraformaldehyde in $0.1 \mathrm{M}$ cacodylate buffer $\mathrm{pH}$ 7.4. Samples were rinsed $3 \times 10 \mathrm{~min}$ in $0.1 \mathrm{M}$ cacodylate buffer at $4{ }^{\circ} \mathrm{C}$ before post-fixation with $1 \% \mathrm{OsO}_{4}$ for $1 \mathrm{~h}$. Samples for EDS measurements were not stained with $\mathrm{OsO}_{4}$. A second rinsing step in $0.1 \mathrm{M}$ cacodylate buffer pH $7.4\left(3 \times 5 \mathrm{~min}\right.$ at $\left.4{ }^{\circ} \mathrm{C}\right)$ was followed by dehydration in ethanol $(50 \%, 70 \%, 90 \%, 3 \times 100 \%)$ for 10 min each step at RT. Threads were embedded in low viscosity Spurr's resin (Electron Microscopy Sciences, \# 14300) at $65^{\circ} \mathrm{C}$ over 2 days. The resulting resin blocks were trimmed to the region of interest and sectioned to $100 \mathrm{~nm}$ using an ultramicrotome (PowerTome Model XL). Ultrathin sections were mounted on lacey carbon coated copper grids (200 mesh) for imaging and EDS measurements.

Transmission electron microscopy. TEM was performed with a Zeiss EM 912 Omega with an acceleration voltage of $120 \mathrm{kV}$ and a Jeol JEM ARM200F equipped with a cold field-emission electron source and a silicon drift detector (SSD), operated at $200 \mathrm{kV}$ acceleration voltage and $15 \mu \mathrm{A}$ emission current. TEM mode was used exclusively for imaging (Bright Field imaging) at magnifications of $10,000 \times$ and $16,000 \times$, whereas STEM mode was used for energy dispersive spectroscopy (EDS) and high angle annular dark field (HAADF) imaging. In STEM mode a fine electron probe scans the surface of the sample pixel-by-pixel enabling identification of the area of the sample that generates certain characteristic X-rays with nanometric resolution. Overview elemental maps of foot sections were acquired at a magnification of 50,000 $\times$ with a pixel size of $17 \mathrm{~nm} \times 17 \mathrm{~nm}$ and an exposure of $1 \mathrm{~s}$ per pixel. For thread sections, elemental maps $(59 \times 89$ pixel $)$ were acquired at a magnification of $200,000 \times$ with a pixel size of $9.3 \times 9.3 \mathrm{~nm}$ and an exposure of $1 \mathrm{~s}$ per pixel using a SDD.

FIB-SEM. Resin blocks containing samples were polished in order to expose the tissue or thread at the block surface. Samples were sputter-coated with three Carbon layers $(\sim 5 \mathrm{~nm}$ each) and one platinum layer $(\sim 5-10 \mathrm{~nm})$ and transferred to the Zeiss Crossbeam 540 (Carl Zeiss Microscopy GmbH, Germany). At the region of interest, a trench for SEM imaging was milled into the sample surface using the $65 \mathrm{nA}$ FIB current at $30 \mathrm{kV}$ acceleration voltage. The resulting cross-section was finely polished using the $1.5 \mathrm{nA}$ FIB probe at $30 \mathrm{kV}$. Measurement of foot tissue and threads required different parameters. Thin slices of samples were removed in a serial manner by FIB milling $(300 \mathrm{pA}, 30 \mathrm{kV}$, slice thickness $17.5 \mathrm{~nm}$ for foot tissue; $700 \mathrm{pA}, 30 \mathrm{kV}$, slide thickness $10.5 \mathrm{~nm}$ for threads). After each milling step, the specimen was imaged by SEM using the secondary and backscattered electron detector (acceleration voltage $=2 \mathrm{kV}$ for foot tissue and $2.5 \mathrm{kV}$ for threads). For foot tissue and threads, the image resolution was $2048 \times 1536$ pixels and $1024 \times 785$ pixels, respectively with a lateral image pixel size of $12.4 \mathrm{~nm}$ and $4.8 \mathrm{~nm}$, respectively. Images were recorded using line averaging $(N=4$ for foot tissue and $N=31$ for threads) and a dwell time of $200 \mathrm{~ns}$.

FIB-SEM data processing. The resulting secondary and back-scattered electron images were processed using the SPYDER3 (Scientific Python Development Environment) (Python 3.6) software. Custom-written python scripts were developed and provided by Luca Bertinetti. For data analysis of cuticle gland tissue, images were automatically aligned using enhanced correlation coefficient alignment. Total variation denoising was performed by applying the Chambolle's projection algorithm (100,000 iterations, weight $0.07,0.001$ eps) in $3 \mathrm{D}$ mode. Segmentation of cuticle vesicles was performed using the ZIB version of Amira 3D (Thermo Fisher Scientific, USA). Vesicle shapes of 28 individual granules were segmented manually from 392 processed back-scattered electron images using the brush tool. The inner phase, which corresponds to the proto-granules as well as the proto-matrix phase were automatically segmented using the Magic Wand tool. 3D visualization of both phases was realized by volume rendering of segmented structures.

For data analysis of the thread cuticle structure, secondary electron images were automatically aligned using the Fourier shift theorem for detecting the translational shift in the frequency domain and vertical stripes arising from the waterfall effect by FIB milling were removed by Fourier filtering. Total variation denoising was performed by applying the Chambolle algorithm in 3D mode. The images were inverted afterwards with Fiji and Sauvola's local thresholding computation was applied with a block size of 11 , a $k$ value of 0.005 and $r$ value of 1.7. As the thresholded images contained regions resulting from statistical noise in the image, only thresholded regions containing minimum 40 pixels were selected. Images were median $3 \mathrm{D}$ filtered with Fiji and $x, y, z$ radii were set to 1.5 . Segmentation of five adjoining cuticle granules was performed using the ZIB version of Amira 3D (Thermo Fisher Scientific, USA). Granule shapes were segmented manually from 352 processed and inverted secondary electron images using the brush tool. In a second step, the lightly staining (ls) granule phase was segmented automatically from local threshold computed and median 3D filtered image stacks using the Magic Wand tool.

Reporting summary. Further information on research design is available in the Nature Research Reporting Summary linked to this article.

\section{Data availability}

The datasets generated during and/or analysed during the current study are available from the corresponding author on reasonable request. 
Received: 30 August 2019; Accepted: 24 January 2020;

Published online: 13 February 2020

\section{References}

1. Sai, H. et al. Hierarchical porous polymer scaffolds from block copolymers. Science 341, 530-534 (2013).

2. Lutz, J. F., Lehn, J. M., Meijer, E. W. \& Matyjaszewski, K. From precision polymers to complex materials and systems. Nat. Rev. Mater. 1, 16024 (2016).

3. Lutolf, M. P. \& Hubbell, J. A. Synthetic biomaterials as instructive extracellular microenvironments for morphogenesis in tissue engineering. Nat. Biotechnol. 23, 47-55 (2005)

4. Webber, M. J., Appel, E. A., Meijer, E. W. \& Langer, R. Supramolecular biomaterials. Nat. Mater. 15, 13-26 (2016).

5. Lehn, J. M. Perspectives in chemistry-steps towards complex matter. Angew. Chem. Int. Ed. 52, 2836-2850 (2013).

6. Lehn, J. M. Toward self-organization and complex matter. Science $\mathbf{2 9 5}$ 2400-2403 (2002).

7. Huo, Q. S., Margolese, D. I. \& Stucky, G. D. Surfactant control of phases in the synthesis of mesoporous silica-based materials. Chem. Mater. 8, 1147-1160 (1996).

8. Zhao, D. Y. et al. Triblock copolymer syntheses of mesoporous silica with periodic 50 to 300 angstrom pores. Science 279, 548-552 (1998).

9. Meseck, G. R., Terpstra, A. S. \& MacLachlan, M. J. Liquid crystal templating of nanomaterials with nature's toolbox. Curr. Opin. Colloid Interface Sci. 29, 9-20 (2017).

10. Fratzl, P. \& Weinkamer, R. Nature's hierarchical materials. Prog. Mater. Sci. 52, 1263-1334 (2007).

11. Degtyar, E., Harrington, M. J., Politi, Y. \& Fratzl, P. The mechanical role of metal ions in biogenic protein-based materials. Angew. Chem. Int. Ed. 53, 12026-12044 (2014).

12. Heim, M., Keerl, D. \& Scheibel, T. Spider silk: from soluble protein to extraordinary fiber. Angew. Chem. Int. Ed. 48, 3584-3596 (2009).

13. Priemel, T., Degtyar, E., Dean, M. N. \& Harrington, M. J. Rapid self-assembly of complex biomolecular architectures during mussel byssus biofabrication. Nat. Commun. 8, 14539 (2017).

14. Baer, A. et al. Mechanoresponsive lipid-protein nanoglobules facilitate reversible fibre formation in velvet worm slime. Nat. Commun. 8, 974 (2017).

15. Winegard, T. et al. Coiling and maturation of a high-performance fibre in hagfish slime gland thread cells. Nat. Commun. 5, 3534 (2014).

16. Denny, M. W. \& Gaylord, B. Marine ecomechanics. Annu. Rev. Mar. Sci. 2, 89-114 (2010).

17. Harrington, M. J., Masic, A., Holten-Andersen, N., Waite, J. H. \& Fratzl, P. Iron-clad fibers: a metal-based biological strategy for hard flexible coatings. Science 328, 216-220 (2010).

18. Holten-Andersen, N., Fantner, G. E., Hohlbauch, S., Waite, J. H. \& Zok, F. W. Protective coatings on extensible biofibres. Nat. Mater. 6, 669-672 (2007).

19. Holten-Andersen, $\mathrm{N}$. et al. Metals and the integrity of a biological coating: the cuticle of mussel byssus. Langmuir 25, 3323-3326 (2009).

20. Schmitt, C. N. Z. et al. Mechanical homeostasis of a DOPA-enriched biological coating from mussels in response to metal variation. J. R. Soc. Interface 12, 20150466 (2015)

21. Monnier, C. A., DeMartini, D. G. \& Waite, J. H. Intertidal exposure favors the soft-studded armor of adaptive mussel coatings. Nat. Commun. 9, 3424 (2018).

22. Lee, B. P., Messersmith, P. B., Israelachvili, J. N. \& Waite, J. H. Musselinspired adhesives and coatings. Annu. Rev. Mater. Res. 41, 99-132 (2011).

23. Li, L., Smitthipong, W. \& Zeng, H. Mussel-inspired hydrogels for biomedical and environmental applications. Polym. Chem. 6, 353-358 (2015)

24. Krogsgaard, M., Nue, V. \& Birkedal, H. Mussel-inspired materials: self-healing through coordination chemistry. Chem. Eur. J. 22, 844-857 (2016).

25. Harrington, M. J., Jehle, F. \& Priemel, T. Mussel byssus structure-function and fabrication as inspiration for biotechnological production of advanced materials. Biotechnol. J. 13, e1800133 (2018).

26. Vitellaro-Zuccarello, L. Ultrastructural and cytochemical study on the enzyme gland of the foot of a mollusk. Tissue Cell 13, 701-713 (1981).

27. Waite, J. H. The formation of mussel byssus: anatomy of a natural manufacturing process. Results Probl. Cell. Differ. 19, 27-54 (1992).

28. DeMartini, D. G., Errico, J. M., Sjoestroem, S., Fenster, A. \& Waite, J. H. A cohort of new adhesive proteins identified from transcriptomic analysis of mussel foot glands. J. R. Soc. Interface 14, 20170151 (2017).

29. Bahr, G. F. Osmium tetroxide and ruthenium tetroxide and their reactions with biologically important substances. Exp. Cell Res. 7, 457-479 (1954).
30. Hayat, M. A. Principles and Techniques of Electron Microscopy. Biological Applications. (Cambridge University Press, Cambridge, 2000)

31. Tamarin, A., Lewis, P. \& Askey, J. Structure and formation of byssus attachment plaque in Mytilus. J. Morphol. 149, 199-221 (1976).

32. Bates, F. S. \& Fredrickson, G. H. Block copolymer thermodynamics-theory and experiment. Annu. Rev. Phys. Chem. 41, 525-557 (1990).

33. Feric, M. et al. Coexisting liquid phases underlie nucleolar subcompartments. Cell 165, 1686-1697 (2016)

34. Holten-Andersen, N. et al. Metal-coordination: using one of nature's tricks to control soft material mechanics. J. Mater. Chem. B 2, 2467-2472 (2014).

35. Creighton, T. E. Proteins: Structures and Molecular Properties. 2nd edn (W. H. Freeman and Company, New York, 1993).

36. Yu, J. et al. Mussel protein adhesion depends on interprotein thiol-mediated redox modulation. Nat. Chem. Biol. 7, 588-590 (2011).

37. Kim, S. et al. Salt triggers the simple coacervation of an underwater adhesive when cations meet aromatic pi electrons in seawater. ACS Nano 11, 6764-6772 (2017)

38. Zhao, H. \& Waite, J. H. Linking adhesive and structural proteins in the attachment plaque of Mytilus californianus. J. Biol. Chem. 281, 26150-26158 (2006).

39. Li, J. Y. \& Li, H. B. Mechanical unfolding pathway of the high-potential ironsulfur protein revealed by single-molecule atomic force microscopy: toward a general unfolding mechanism for iron-sulfur proteins. J. Phys. Chem. B 122, 9340-9349 (2018)

40. Lee, H., Scherer, N. F. \& Messersmith, P. B. Single-molecule mechanics of mussel adhesion. Proc. Natl Acad. Sci. USA 103, 12999-13003 (2006).

41. George, S. G., Pirie, B. J. S. \& Coombs, T. L. The kinetics of accumulation and excretion of ferric hydroxide in Mytilus edulis and its distribution in the tissues. J. Exp. Mar. Biol. Ecol. 23, 71-84 (1976).

\section{Acknowledgements}

The authors acknowledge funding from the Max Planck Society and the German Research Foundation (DFG individual grant HA 6369 5). M.J.H. acknowledges support from the Natural Sciences and Engineering Research Council of Canada (NSERC Discovery Grant RGPIN-2018-05243).

\section{Author contributions}

M.J.H., L.B. and P.F. devised and oversaw the study. L.B., F.J., S.S. and E.M.-S. performed experiments and processed data. M.J.H. and F.J. wrote the paper. All authors contributed to editing the paper.

\section{Competing interests}

The authors declare no competing interests.

\section{Additional information}

Supplementary information is available for this paper at https://doi.org/10.1038/s41467 020-14709-y.

Correspondence and requests for materials should be addressed to L.B. or M.J.H.

Peer review information Nature Communications thanks Emily Carrington and the other anonymous reviewer(s) for their contribution to the peer review of this work.

Reprints and permission information is available at http://www.nature.com/reprint

Publisher's note Springer Nature remains neutral with regard to jurisdictional claims in published maps and institutional affiliations.

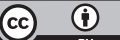

Open Access This article is licensed under a Creative Commons Attribution 4.0 International License, which permits use, sharing adaptation, distribution and reproduction in any medium or format, as long as you give appropriate credit to the original author(s) and the source, provide a link to the Creative Commons license, and indicate if changes were made. The images or other third party material in this article are included in the article's Creative Commons license, unless indicated otherwise in a credit line to the material. If material is not included in the article's Creative Commons license and your intended use is not permitted by statutory regulation or exceeds the permitted use, you will need to obtain permission directly from the copyright holder. To view a copy of this license, visit http://creativecommons.org/ licenses/by/4.0/.

(c) The Author(s) 2020 